\title{
Height-Relative Determination of (Non-Root) Modal Flavor: Evidence from Hindi*
}

\author{
Dave Kush \\ University of Maryland
}

\begin{abstract}
In this paper it is proposed that modal flavor is determined by the attachment height of a modal. The various interpretations of the Hindi future marker gaa, which is taken to be a modal, are discussed. The idea put forth is that modal flavor is indirectly constrained by the semantic type of the modal's prejacent instead of being solely determined via contextual assignment. Modal Bases are re-envisioned as being comprised of different types of alternatives (worlds, world-time pairs, etc.), rather than just sets of worlds determined by different accessibility relations. The correlation between height and attachment site falls out as a consequence of the different types of alternatives Modal Bases make available for semantic computation.
\end{abstract}

Keywords: Modality, Epistemic, Future, Hindi, Height-Relativity

\section{Height Relativity of Modal Flavor}

It has been observed that a modal's attachment height seems to correlate with its interpretation (Brennan 1993, Cinque 1999, Hacquard 2010, Jackendoff 1972, a.o.). With root interpretations, modals appear to scope low (1), while epistemic modals have been argued to scope above Tense (2).

(1) John had to make it home before his mother.

(2) John had to be home (at the time of the crime).

(Examples adapted from Hacquard 2010)

In (1), John's obligation is in the past, the time set by Tense. In (2), the modal is said to scope above Tense. This is because, despite the past-marked verb, the time at which the evidence for the epistemic claim is evaluated is the speech time.

In this paper, I note that a similar height-interpretation correlation exists in the non-root domain. Namely, a (particular Hindi) modal's position determines whether it is treated as a future-marker or as an epistemic modal.

* Thanks first and foremost to Valentine Hacquard, Norbert Hornstein, Alexander Williams for extensive discussion of this work. Thanks as well to Rajesh Bhatt, Ashwini Deo and Ashok Kush for helpful insights. Members of the CNL Lab at UMD also deserve thanks for comments and feedback. All errors are mine. Thanks also to the organizers of SALT. This work was funded, in part, by an NSF IGERT training grant (DGE-0801465).

(C)2011 Dave Kush 
While many authors have noted the correlation between height and root/epistemic flavor, few have attempted to derive it. ${ }^{1}$ I explore the idea that modal flavor is indirectly determined by the semantic type of a modal's syntactic sister. The roots of this idea can be traced back to Brennan 1993, who argued that the interpretation of various modals was sensitive to their complements.

Unlike Brennan, I argue for a type-driven analysis of the correlation. Contrary to the traditional view, I argue that Modal Bases can be sets of more than just worlds. Instead, they are re-envisioned sets of tuples, whose make-up varies with the different accessibility relation. Thus, for every Modal Base there is a unique tuple. The position of the modal effects Modal Base-selection by constraining the kind of tuple the modal quantifies over, in accordance with the Type-Alternative Correspondence (3).

(3) Type-Alternative CoRrespondence

A modal, $\mathscr{M}$, quantifies over alternatives that are tuples $(a, b, \ldots)$, where the sister of $\mathscr{M}$ is of type $\langle\mathscr{A},\langle\mathscr{B},\langle\ldots, t\rangle\rangle\rangle$ and types $\mathscr{A}, \mathscr{B} \ldots$ are the corresponding types of variables $a, b$, etc.

The paper has the following structure. Section 2 presents the core data from Hindi that the paper is concerned with. Section 3 provides a brief overview of Kratzer's $(1981,1991)$ treatment of modals and the Modal Bases. Section 4 presents evidence for the height-interpretation correlation in Hindi. Section 5 considers how the idea of a Modal Base must be revised. Section 6 briefly discusses how the account presented here derives the correlation. Section 7 discusses possibly extending the account to root modals as well. Section 8 concludes.

\section{Hindi $g a a$}

The Hindi future-marker gaa can be used to make both Plain Future (4) and Epistemic (5) statements (Sharma 2008).

(4) Amitabh kaam kar-e-gaa

Amitabh work do-Subj-gaa

'Amitabh will do work.'

(5) Amitabh kaam kar-taa ho- $\varnothing$-gaa

Amitabh work do-IMPF aux.Subj-gaa

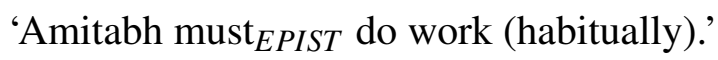

1 One recent exception to this claim is Hacquard 2010, who proposed an event-relative account of height-flavor correlation. 
Height-Relativity of Modals

Importantly, the readings of gaa are constrained. For the purposes of this paper, I maintain that a complementarity exists in availability of readings with gaa. The Epistemic reading is impossible in the absence of an auxiliary (6). When gaa appears on a Tense auxiliary, the future reading is blocked. ${ }^{2}$

(6) Amitabh kaam kar-e-gaa

Amitabh work do-Subj-gaa

\#‘Amitabh must $E P I S T$ do work.'

(7) Amitabh kaam kar-taa ho- $\varnothing$-gaa

Amitabh work do-IMPF aux.Subj-gaa

\#'Amitabh will do work (habitually).'

\section{Modal Bases}

Kratzer $(1981,1991)$ outlined an influential theory in which contextual backgrounds determine the interpretation of a modal. The contextual background relevant to the analysis proposed here is the Modal Base (MB). ${ }^{3}$ MBs are sets of worlds (or functions from worlds to propositions) determined by a particular accessibility relation. Different modal flavors correspond to different MBs. For example, epistemic modals take the EPISTEMIC MB. Root modals (dynamic modals, deontics, etc.) take the Circumstantial MB.

(8) EPISTEMiC MB

The set of worlds consistent with what is known in $\mathrm{w}^{*}$ (at $\left.\mathrm{t}^{*}\right)$.

(9) Circumstantial MB

The set of worlds consistent with circumstances in $\mathrm{w}^{*}$.

According to Kratzer, modals like may have only a single entry in the lexicon, with an unspecified MB parameter. The apparent difference of meaning for may in (10a) and (10b) can be attributed to differences in MB.

a. John may play in the basement (since he has finished his homework). DEOnTIC - CiRCumstantial MB

John may be playing the basement (for all I know, since I haven't seen him for hours). EPISTEMIC - EPISTEMIC MB

2 The data are, in fact, slightly more complicated than I have made them out to be. However, I do believe this is the right characterization and defend it at greater length in Kush (Submitted), where I also consider progressive and perfect constructions which apparently allow Future and Epistemic readings, despite having an overt auxiliary.

3 The Ordering Source was also an important component of Kratzer's take on modals, but my account here has nothing to say about them. 
In this paper I adopt the Kratzerian view that differences in modal flavor arise due to differences in MB. However, I depart from Kratzer in that I take the procedure by which MB is determined to be structurally, rather than contextually, determined.

\subsection{Metaphysical Modality}

The Kratzerian picture of MBs has been recently expanded to cover readings above and beyond the original epistemic/root distinction. Condoravdi (2002) introduces a new MB, the METAPHYSICAL MB, that she uses for future (and counterfactual) interpretations. Informally, the Metaphysical MB is based on the notion that possible futures branch forward from a particular evaluation time. In the diagram below, 3 futures branch from the present $\left(\mathrm{t}^{*}\right)$; each branch is a world, a metaphysical alternative.

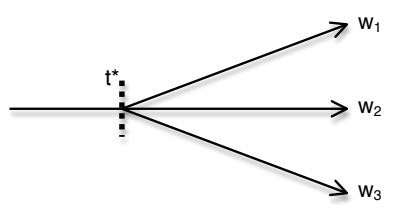

Figure 1 Possible Futures Branching from $t^{*}$

The Metaphysical MB, on Condoravdi's account, can be thought of as the set of the worlds (future branches) that project from a certain point in time. This branching point is the Evaluation Time of the modal.

(1b) MetaphysicAl MB

The set of possible futures branching from $t$.

Having familiarized the reader with the Metaphysical MB, we can move on to the position of gaa.

\section{Height of Hindi gaa}

In this section I show that gaa attaches in two positions: sister to TP and sister to AspP. These positions correlate with interpretation.

- $[[g a a+T P]] \rightsquigarrow$ Epistemic

- $\quad[$ gaa + AspP $] \rrbracket \rightsquigarrow$ Future

Determining gaa's position vis-a-vis Tense is crucial to determining its height. 
Height-Relativity of Modals

\subsection{No Tense with Futures}

Following Anand \& Nevins (2007) and Davison (2002) I take the auxiliary ho (and its various forms) to be a spell-out of Tense.

(13) Amitabh kaam kar-taa hai/thaa.

Amitabh work do-IMPF aux-PRES/PAST

'Amitabh works/used to work.'

From the ungrammaticality of auxiliaries in Future constructions we can conclude that Tense is absent.
a. Amitabh kaam kar-e-gaa.
Amitabh work do-Subj-gaa.
'Amitabh will do work.'
b. *Amitabh kaam kar ho-gaa.
Amitabh work do aux-Subj-gaa.
c. Amitabh kaam kar-e-gaa ho.
Amitabh work do-Subj-gaa aux.

Given the absence of Tense with Future constructions, I assume that Future gaa must sit directly above AspP.

\subsection{Tense Required with Epistemics}

In contrast to Future constructions, Epistemic constructions must have Tense. We begin by noting that the Evaluation Time of gaa is set by a commanding operator. In the example below, gaa's Evaluation Time is keyed to the attitude time of the commanding verb soc-aa (thought). Were it not, the sentence would be contradictory.

(15) Amitabh-ne soc-aa thaa ki Saif aam pasand kar-taa

Amitabh-ERG think-PFV aux.PAST C Saif mango like do-IMPF ho-gaa, par abhi voh jan-taa hai ki yeh galat thaa. aux-gaa, but now 3sg know-IMPF aux.PRES C this wrong aux.PAST

'Amitabh thought that Saif must eat mangoes, but now he knows that's wrong.'

Next, note that the Instantiation Time of Epistemic gaa can be back-shifted with the use of an appropriate adverbial phrase. One can utter the sentence below, which is an epistemic claim about a particular past action of Ramu's.

(16) (After coming across a CD from 1995 on which Ramu, a man whose hands were cut off in 2000, is credited as a jazz pianist.) 
Dave Kush

Haath kaTne se pehele, Raamu piano bajaa-taa ho-gaa.

Hand cut from before, Ramu piano play-IMPF aux-gaa

'Before cutting off his hands, Ramu must have played piano.'

The adverbial alone cannot be the locus of the back-shift, since adverbials must agree with Tense.

(17) Haath kaTne se pehele, Raamu piano bajaa-taa *hai $/ \checkmark$ thaa.

Hand cut from before, Ramu piano play-IMPF aux-*PRES/PAST

Moreover, the back-shift cannot be due to the presence of a Perfect Operator because Imperfective Morphology is incompatible with perfect interpretation.

a. Amitabh yeh baat (\#hamesha) jaan-taa hai.

Amitabh this thing (\#always) know-IMPF aux.PRES.

'Amitabh knows that.' $\neq$ 'Amitabh has always known that.'

b. \#Picchle saaloN me, Amitabh mumbai me reh-taa hai.

Last years in, Amitabh Bombay in live-IMPF aux.PRES.

\#‘For the last few years, Amitabh has been living in Bombay.' (Tests from Iatridou, Anagnostopoulou \& Izvorski 2001)

Thus, Tense must be present in Epistemic constructions. Given this, it behooves us to determine Epistemic gaa's position with respect to Tense. Recall that Epistemic gaa's Evaluation Time is set by a commanding operator (shown in (15)). We see below that the Evaluation Time of Epistemic gaa in (16) is immune to back-shifting.

(19) Haath kaTne se pehele, Raamu piano bajaa-taa ho-gaa, (\#par maiN Hand cut from before, Ramu piano play-IMPF aux-gaa, but $1 \mathrm{sg}$ abhi jaan-taa huN ki us-NE aisa nahiiN kar-taa thaa). now know-IMPF aux.PRES C 3sg-ERG so NEG do-PFV $\neq$ 'It must have been the case that before cutting off his hands Raamu used to play piano, (\#but now I know that's not true).'

Based on this, we can conclude that Epistemic gaa sits above Tense, for its Evaluation Time would otherwise be back-shifted. Positioning epistemic gaa above $\mathrm{T}$ is consistent with cross-linguistic observations made by researchers such as Cinque (1999), and the data discussed in (2).

The proposed structures are below: 
Height-Relativity of Modals

(20) FUTURE

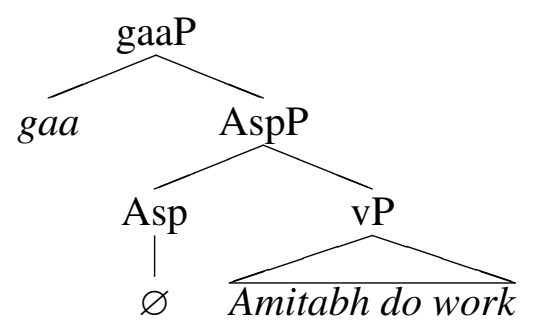

(21) EPISTEMIC

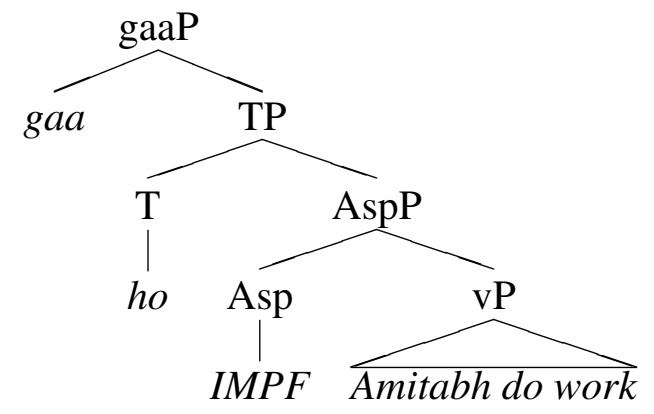

\subsection{Denotation of gaa}

We've seen that gaa can appear in two positions, and that there is a different interpretation associated with each of these positions. One possible solution would be to simply list two distinct gaas in the lexicon, each with its interpretation hardwired. However, this would go against the Kratzerian intuition that lexical ambiguity should be avoided for modals. Rather, it would be preferable to have one gaa.

To appear in multiple positions, gaa receives a flexible type. Its core lexical entry employs a meta-variable $\alpha$ that stands in for kinds of alternative tuples:

$$
\left[[g a a]^{g, c}=\lambda P . \lambda t . \lambda w . \forall \alpha \in \mathrm{MB}(\mathrm{w}, \mathrm{t}) \rightarrow \mathrm{P}(\alpha)\right.
$$

Values of $\alpha$ differ, depending on the sister's semantic type, following the TYPEAlternative CORRESPONDENCE. Below are the two expansions of gaa's denotation, depending on its height:

$$
\left[[g a a]^{g, c}=\left\{\begin{aligned}
\lambda p_{\langle s t\rangle} \cdot \lambda t . \lambda w . \forall\left(\mathrm{w}^{\prime}\right) \in M B(\mathrm{w}, \mathrm{t}) \rightarrow \mathrm{p}\left(\mathrm{w}^{\prime}\right) & (\text { when sister is TP }) \\
\lambda P_{\langle i, s t\rangle} \cdot \lambda t \cdot \lambda w . \forall\left(\mathrm{w}^{\prime}, \mathrm{t}^{\prime}\right) \in M B(\mathrm{w}, \mathrm{t}) \rightarrow \mathrm{P}\left(\mathrm{w}^{\prime}, \mathrm{t}^{\prime}\right) & (\text { when sister is AspP) }
\end{aligned}\right.\right.
$$

It is important to note that nothing in gaa's denotation specifies the height-flavor correspondence. Its MB parameter is left unspecified. In section 6 I explain how this constraint is achieved. In order to do that, however, the traditional conception of what can constitute an MB must first be altered. 


\section{Recasting Modal Bases}

Modal bases are traditionally treated as sets of worlds (or functions from worlds to propositions). In this section I'd like to propose that while some MBs may be sets of worlds, others are different. Specifically, I propose that the Metaphysical MB is comprised of world-time pairs. The Epistemic MB is, as on Kratzer's account, still a set of worlds.

While the Metaphysical MB is the dedicated MB for future readings in accounts like Condoravdi's, its association with future orientation is often arrived at indirectly. In the Condoravdian system, present- and past-oriented uses of modal expressions keyed to the Metaphysical MB are ruled out by a Diversity Condition on modal utterances. Despite the fact that the use of the MB is restricted to future-oriented utterances, the modal itself is the locus of future-orientation. This, in principle, allows for future-oriented epistemic claims, contrary to fact.

I propose that we treat the Metaphysical MB differently from the epistemic MB. Epistemic modality is dissociable from temporal perspective. The temporal orientation of an epistemic modal's prejacent can either be past- or present-oriented. Metaphysical modals are restricted to future-orientation.

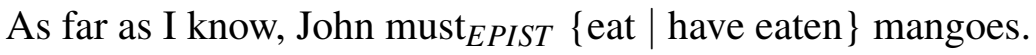

a. John will METAPHYSICAL go to the store (*yesterday).

b. John said on Friday that he would ${ }_{\text {METAPHYSICAL }}$ go to the store (last $\{*$ Thursday $\mid$ Saturday $\}$ )

Epistemic and Metaphysical MBs differ in the role of a modal's Evaluation Time vis-a-vis its Instantiation Time. An Epistemic modal's Evaluation Time and Instantiation Time are distinct. This was seen in (2), where the action described by the verb took place in the past, but the evidence used to evaluate the epistemic claim is relative to the speech time. A Metaphysical modal's Evaluation Time, on the other hand, partially determines the modal's Instantiation Time. The Instantiation Time of a Metaphysical modal is always subsequent to the Evaluation Time ( $t^{*}$ in plain Futures).

All of this is to say that Metaphysical modality and future temporal reference are inextricably linked. I propose that we should make this interdependence explicit, and separate the Metaphysical MB from other MBs in terms of the types of alternatives it ranges over. I suggest that, rather than a world, each branch represents a world-time pair $(\mathrm{w}, \mathrm{t})$, where the temporal argument is some time on the future branch. ${ }^{4}$ To characterize the set:

4 It is also possible that the time argument supplied by the MB as the open-ended interval $\left(t^{*}, \infty\right)$. The analysis chosen will have obvious consequences for the denotations of aspectual operators, but these consequences are largely irrelevant to the point of the current paper. 
Height-Relativity of Modals

(26) MetAphysicAl MB

The set of $(\mathrm{w}, \mathrm{t})$ pairs such that $\mathrm{w}$ is a Metaphysical alternative $\mathrm{w}^{*}$ and $\mathrm{t}^{\prime}>$ $t^{*}$

The $t$ ' supplied by the MB is used to saturate the time argument of the prejacent that gaa takes as its complement. Unlike Epistemic sentences, which receive their temporal specification from Tense, Futures receive their temporal argument from the Metaphysical MB.

The distinction between the types of alternatives that the MBs quantify over is the crux of my analysis and the explanation for the interpretive restrictions discussed in section 2. By definition, the Metaphysical MB quantifies over future-oriented alternatives. By quantifying over Metaphysically accessible alternatives, the modal obtains its future orientation.

\section{Restricting Flavor}

In section 4.3 I argued that the flavor restrictions need not be encoded in the lexicon. Here I show how the restrictions fall out from the TAC and the reformulation of MBs.

We have seen that gaa can attach in two positions, and that these positions fix the kinds of alternatives gaa can quantify over. TP is $\langle s t\rangle$, having an unsaturated world argument. Thus, according to the TAC gaa is restricted to quantifying over worlds when it has a TP sister. AspP is $\langle i, s t\rangle$, with unsaturated time and world arguments, therefore gaa quantifies over $(\mathrm{w}, \mathrm{t})$ pairs when it has an AspP sister. The basic idea pursued here is that by positionally fixing gaa's alternatives, we limit the compatible MBs.

When gaa composes with a property of times (AspP), Epistemic modality is blocked. In that position gaa necessarily quantifies over world-time pairs. Because the Epistemic MB is a set of worlds, not world-time pairs, it is not eligible for selection. The only MB with the appropriate kind of alternatives is the Metaphysical.

The opposite is true when gaa takes TP as its sister. Gaa can only take an MB that provides alternative worlds. As such, the Metaphysical MB is not eligible, and the Epistemic MB is. ${ }^{5}$

5 Gaa cannot contribute root modality. If the Circumstantial MB is also a set of worlds, nothing in the account would prevent its selection. There are two options: (i) suppose that the gaa is lexically barred from taking the Circumstantial MB, or, (ii) suppose that the Circumstantial MB is not a set of just worlds, after all. The latter option is considered at the end of the paper. 


\section{Extension to Root Modals?}

Above I sketched a height-relative, type-driven account of the interpretation of Hindi gaa. The analysis was only concerned with deriving the Epistemic and Metaphysical readings of the modal. However, as noted at the outset, traditional discussions of height-relativity centered around the root/epistemic split. It is therefore natural to ask whether the type-driven account outlined above could be extended to root modals. The answer is a qualified yes.

The extension to root modals starts from the observation that such modals are 'subject-oriented', as opposed to Epistemics which are speaker-oriented (Jackendoff 1972, a.o.). From the perspective of altering the MB, extending the account to circumstantial modality is straightforward. We could recast the CIRCUMSTANTIAL MB along the following lines:

Circumstantial MB:

The set of $(\mathrm{x}, \mathrm{w})$ pairs such that $w$ is a world compatible with facts about $x$ in $\mathrm{w}^{*} \cdot 6$

Specifying a syntax for such constructions, on the other hand, is a trickier proposition.

A root modal will require an $\langle e,\langle s t\rangle\rangle$ complement. Yet, on standard assumptions about the $\mathrm{v} / \mathrm{V}$ domain, there are no maximal phrases that meet this requirement. Neither VP nor vP are suitable candidates for the sister of a root modal (without auxiliary assumptions), since they are $\langle s, t\rangle$.

One potential proposal hearkens back to Jackendoff 1972 and Ross 1969, both of which treated root modals as types of control structures. Let's consider the idea that root modals take vP sisters, but that the specifier of vP must be PRO. Since PRO must be bound, this solution would require the subject position to be abstracted over, creating a complement of the required type. The binder of the PRO would be introduced in a phrase immediately above ModP, XP for now, as shown below.

6 Note that I am not committed to the exact formulation of the relation between $x$ and $w$, so long as the $\mathrm{MB}$ is constituted of $(\mathrm{x}, \mathrm{w})$ pairs. 
Height-Relativity of Modals

(28)

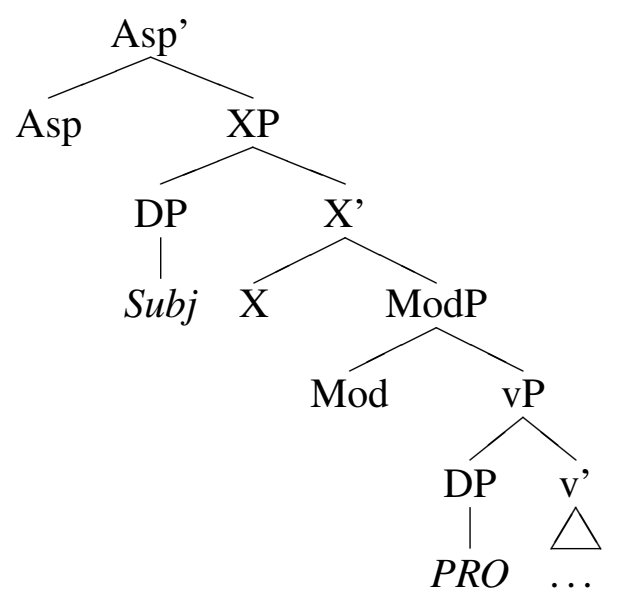

While I have used XP as the phrase that would introduce the binder of PRO, one possibility is that it is simply another vP. This treatment would entail treating root modals as verbs that take infinitival complements (as Ross 1969 did). However, there are problems with this kind of analysis. In previous work many arguments against the control analysis of modal constructions have been presented; I refer the reader to Palmer (1979), Brennan (1993) and Wurmbrand (1999) for discussion of these. One problem discussed in Wurmbrand (1999) is that a thematic relation is not always required between the subject and the modal in some root modal constructions.

(29) There can be a party as long as it's not too loud. (Wurmbrand 1999: ex. 20a)

The control analysis of root modals cannot handle this data point. It is clear that this syntactic treatment raises many questions, not the least of which is concerned with the licensing of the external argument, that I do not solve here. Despite this, I believe it is an interesting first step in assessing whether the type-driven analysis could be given to all modal interpretations uniformly.

\section{Conclusion}

In this paper I outlined a type-driven, height-relative account of non-root modal interpretation based on evidence from the Hindi modal gaa. It was proposed that Metaphysical modals (future-markers) take AspPs as their sisters, whereas Epsitemic modals take TPs. The kinds of alternatives that the modal's denotation allowed for were determined by the modal's position, in accordance with the TYPEAlternative Correspondence. Modal Bases were reconfigured so that each Modal Base received a unique alternative type. Extending the analysis to root modality was also considered, though the syntactic details of such an extension were not developed in great detail. For a more detailed discussion of the account, the 
phenomena discussed above, and other related issues, the reader is referred to Kush Submitted.

\section{References}

Anand, Pranav \& Andrew Nevins. 2007. The locus of ergative case assignment: Evidence from scope. In Ergativity: Emerging issues, Berlin: Springer Verlag.

Brennan, Virginia. 1993. Root and epistemic modal auxiliaries: UMASS-Amherst dissertation.

Cinque, Guglielmo. 1999. Adverbs and functional heads: a cross-linguistic perspective. New York: Oxford University Press.

Condoravdi, Cleo. 2002. Temporal interpretation of modals, vol. The Construction of Meaning, 59-88. Stanford, CA: CSLI Publications.

Davison, Alice. 2002. Agreement features and projections of tense and aspect. In R. Singh (ed.), Yearbook of South Asian languages and linguistics, 27-57. Sage Publications.

Hacquard, Valentine. 2010. On the event-relativity of modal auxiliaries. Natural Language Semantics 18(1). 79-114.

Iatridou, Sabine, E. Anagnostopoulou \& Roumyana Izvorski. 2001. Observations about the form and meaning of the perfect. In M. Kenstowicz (ed.), Ken Hale: A life in language, MIT Press.

Jackendoff, Ray. 1972. Semantic interpretation in generative grammar. Cambridge, Massachusetts: MIT Press.

Kratzer, Angelika. 1981. The notional category of modality. In H.-J. Eikmeyer \& H. Rieser (eds.), Words, worlds, and contexts. new approaches in word semantics, De Gruyter.

Kratzer, Angelika. 1991. Modality. In A. von Stechow \& D. Wunderlich (eds.), Semantik: Ein internationales Handbuch zeitgenoessischer Forschung, 639-650. Berlin: De Gruyter.

Kush, Dave. Submitted. The future of Hindi modality. ms., University of Maryland. Palmer, F. R. 1979. Why auxiliaries are not main verbs. Lingua 47. 1-25.

Ross, John. 1969. Auxiliaries as main verbs. In Studies in Philosophical Linguistics, Evanston, IL: Great Expectations Press.

Sharma, Ganshyam. 2008. A pragmatic account of the hindi presumptive. Annual Review of South Asian Languages and Linguistics, 83-116.

Wurmbrand, Susi. 1999. Modal verbs must be raising verbs. In S. Bird, A. Carnie, J. Haugen \& P. Norquest (eds.), WCCFL 18 Proceedings, 599-612. Somerville, MA: Cascadilla Press. 
Height-Relativity of Modals

Dave Kush

University of Maryland

1401 Marie Mount Hall

College Park, MD 20742

kush@umd.edu 